\title{
EMPLEABILIDAD DE EGRESADOS DE LA FACULTAD DE CIENCIAS ADMINISTRATIVAS DE LA UNMSM
}

\author{
Pedro L. Tito Huamaní (") \\ E-mail: pedrotito18@hotmail.com \\ Fabiola Pereda Lévano ${ }^{(*)}$ \\ E-mail:fpereda07@gmail.com \\ Patricia Vilcabana Noriega ${ }^{(-m)}$ \\ E-mail: pavilcabana@hotmail.com
}

\begin{abstract}
RESUMEN
Los resultados de la investigación revelan que existe una mediana correspondencia entre el ámbito de ocupación laboral definido por la universidad, a través de sus planes de estudios, y el nivel de empleabilidad y ocupabilidad de nuestros egresados en el mercado laboral. Tal afirmación se corrobora cuando el 53\% de los encuestados manifestaron que los conocimientos impartidos en la universidad sirvieron poco o medianamente para su desempeño laboral óptimo. Ello implica que la Facultad de Ciencias Administrativas de la Universidad Nacional Mayor de San Marcos (UNMSM) debe mantener una comunicación estrecha con los stakeholders, a fin de saber cuáles son sus requerimientos en términos de recursos profesionales, para incorporarlos en sus futuros planes de estudios
\end{abstract}

Asimismo, la investigación también demuestra que un porcentaje representativo de nuestros egresados tienen empleos en posiciones inferiores dentro de la estructura organizacional, cumpliendo funciones administrativas y operativas poco o nada gravitantes para la organización. Esta situación se puede explicar desde dos posiciones: a) Que los encuestados fueron en su mayoría egresados de los últimos años (2004-2006), que recién se inician profesionalmente a laborar, y es obvio que lo harán desde abajo, y b) Que los planes de estudios no guardan relación con lo que exige el mercado laboral, por lo que nuestros egresados sistemáticamente son postergados de ascender a funciones o puestos relevantes, dado que no se encuentran debidamente preparados para ello. Contrastando y cruzando otros datos, podemos afirmar que los empleos poco relevantes de nuestros egresados se explican por la primera posición, lo que a su vez demuestra un elemento incontrolable en la investigación.

(*) Doctorado en Ciencias Administrativas, Magíster en Gestión Empresarial y Licenciado en Administración (UNMSM). Docente permanente y docente de Postgrado (UNMSM). Profesor visitante de las universidades UNP (Piura), UNT (Trujillo), UNLG (Ica), UNA (Puno) y UNSCH (Ayacucho). Consultor en Gestión Humana y Comportamiento Organizacional.

(***) Licenciada en Administración. UNMSM.

(***) Bachiller en Ciencias Administrativas. UNMSM. 
En ese orden de ideas, la preocupación por el nivel de empleabilidad y ocupabilidad de nuestros egresados debe ser uno de los objetivos principales de la Facultad; ello implica que al momento de reestructurar los planes de estudios, en la fase de diagnóstico, se deben tomar muy en cuenta las opiniones y sugerencias que emitan los líderes y directivos de las organizaciones privadas o públicas.

Debemos entender que la empleabilidad es un concepto mucho más dinámico que el empleo, puesto de trabajo o capacitación profesional. Trabajar por la empleabilidad de nuestros egresados implica potenciar sus competencias para conseguir trabajos y conservarlos; esto es mucho más que el proceso que conduce solo a la obtención del Título Profesional.

En ese contexto, la UNMSM debe proporcionar un balance entre la formación técnica y científica para trabajos especializados, sin descuidar la formación del ciudadano que se integra a la sociedad para trascender en el tiempo. Postulamos, en suma, a una empleabilidad con formación integral del hombre que conjugue lo conceptual con lo procedimental y lo actitudinal, fundamentalmente.

Palabras Clave: Empleabilidad, Egresados, Plan de Estudios, Perfil Profesional, Oferta Laboral, Demanda Laboral.

\begin{abstract}
The results of this research reveal that exist a medium correspondence among the environment of labor occupation defined by the university through its studies plans, and the level of employability and occupation of our graduates in the labor market. Such affirmation is corroborated when 53\% of the polled declared that the know-how given in the University served a little or moderately for their optimum labor performance. It implies that the Faculty of Administrative Sciences of our University (UNMSM) should maintain a narrow communication with the stakeholders, in order to know which are their requirements in terms of professional resources to incorporate them in their future studies plans.
\end{abstract}

Likewise, the investigation also shows that a representative percentage of our graduates have jobs in lower positions inside the structure organizacional, complying operating and administrative functions little or menacing nothing for the organization. This situation can be explained since two positions: a) That the polled were mostly graduates of recent years (2004-2006), that recently initiated professionally to labor, and is obvious that will do it from below, and b) That the plans of studies do not keep relation with what requires the labor market, for which our graduates systematically are deferred to elevate to functions or prominent positions, given that they are not found properly prepared for it. Contrasting and crossing other data, we can affirm that prominent little jobs of our graduates are explained for the first position, and at the same time shows an uncontrollable element in the investigation.

In that order of ideas, the worry by level of employability and occupation of our graduates should be one of the main objectives of the Faculty; it implies that in the moment of restructure the plans of studies in the phase of diagnosis, they should be taken all the opinions and suggestions that emit the leaders and executives of the private or public organizations.

We should understand that the employability is an a lot more dynamic concept than the employment, job or professional training. Working for the employability of our graduates implies to promote its competences to obtain works and to conserve them; this is a lot more than the process that conducts only to obtaining the Professional Title.

In that context, the UNMSM should provide a balance between the technical formation and scientist for specialized works, without neglecting the formation of the citizen that is integrated to the company to transcend in the time. We advance in short to employability with integral formation of the man, that conjugates fundamentally the conceptual thing with the procedural thing and it attitudinal.

Key words: Employability, Graduates, Studies Plan, Professional Profile, Labor Offering, Labor Demand 


\section{INTRODUCCIÓN}

El presente trabajo busca analizar y diagnosticar el nivel de empleabilidad y ocupabilidad de los egresados de la Facultad de Ciencias Administrativas de la Universidad Nacional Mayor de San Marcos (UNMSM). Para ello, se ha utilizado como método de investigación la recolección de datos, aplicando una encuesta ad hoc a todos los bachilleres de la Facultad que reiniciaron sus trámites en las diversas unidades académicas y administrativas de la Facultad para optar sus Títulos Profesionales. Cabe recordar que en el periodo de realización de estudios (año 2007), las Escuelas Académicos Profesionales de la Facultad (Administración, Administración en Negocios Internacionales y Administración en Turismo), desarrollaron cursos de actualización que condujeron al otorgamiento de Títulos Profesionales. Así los datos obtenidos, en su segunda fase, fueron corroborados, desechados o en su defecto precisados, a través de entrevistas que se realizaron a los bachilleres en forma aleatoria.

La presente investigación tuvo como propósito central obtener información primaria para verificar si, en efecto, existe correspondencia entre los planes de estudios con lo que requiere el mercado laboral, constituido por las empresas, instituciones públicas u organismos mixtos. A la fecha, los resultados vienen sirviendo de insumo para retroalimentar de información el diseño de los planes curriculares que la Facultad realiza con regularidad, con la finalidad de potenciar las competencias de los próximos egresados con los conocimientos, habilidades, destrezas y actitudes concordantes con la demanda laboral.

La recopilación de los datos a través de la aplicación de la encuesta fue la más tediosa, dado que nuestro trabajo estuvo condicionado a la lenta reincorporación de los bachilleres. Una vez que se contrastaron los resultados, pensamos que este detalle fue una limitante en nuestra investigación pues, a nuestro modo de ver, la edad y los años de egresado son determinantes para realizar funciones importantes en la especialidad; vale decir, cuanto mayor sea la edad y los años de egresado, mayores serán los niveles de empleabilidad en puestos y cargos más relevantes de la organización, a diferencia de aquellos que tienen pocos años de egresados.

\section{MARCO TEORICO}

Con el propósito de validar nuestro planteamiento fue necesario revisar la información que proviene de fuentes secundarias. Los comentarios de los siguientes autores que realizaron investigaciones similares o afines nos sirven en esta ocasión.

El profesor Nemesio Espinoza (2004) realizó una investigación entre los estudiantes de Administración de las universidades públicas de Lima (UNMSM, UNFV y UNC) ${ }^{1}$ con el propósito de medir sus aptitudes y actitudes empresariales. El autor se plantea como objetivo de estudio conocer las causas y características del desempleo profesional en el Perú, así como las estrategias educativas vigentes en tales facultades a esa fecha, y las repercusiones de las actitudes y aptitudes empresariales en los estudiantes de Administración. Para el trabajo de campo se aplicaron cuestionarios a estudiantes de diferentes ciclos, edades y turnos de estudio.

El profesor Espinoza, a modo de conclusión de la investigación, manifiesta que

“...se aprecia la continuidad -como hace décadas- de las mismas estrategias formativas orientadas a formar profesionales en Administración, para que ocupen cargos gerenciales en las grandes empresas $y$, ante la imposibilidad de tal ocurrencia, los egresados, irremediablemente, conforman un gran contingente de administradores sin empleo. No existen estrategias pedagógicas orientadas a formar empresarios ni a promover aptitudes y actitudes empresariales".

Argumenta que una estrategia importante para revertir esta incoherencia entre lo que exige el mercado versus lo que forman las universidades es el

“...cambio de paradigmas educativos en las facultades de Administración de las universidades públicas a fin de que están orientadas más a la formación de empresarios y a la inculcación sistemática de las actitudes y aptitudes empresariales en los estudiantes durante su formación profesional".

Otra fuente importante que se analizó fue el estudio desarrollado por la Universidad Politécnica de Cartagena (Colombia) ${ }^{2}$, referida a la carac-

1 Espinoza, Nemesio. (2004). 
terización y empleabilidad de los egresados de la Facultad de Ciencias de la Empresa, planteándose como objetivos de la investigación

“...describir y analizar la situación de empleabilidad de los egresados de la Facultad de Ciencias de la Empresa de la Universidad Politécnica de Cartagena en los cursos académicos 2001/02 y 2002/03".

Hay plena coincidencia con la utilidad de la información generada porque, al igual que en nuestra investigación, sus resultados sirvieron como insumos importantes para la toma de decisiones de los directivos de la mencionada universidad.

La investigación destaca el número de años que los egresados emplean para obtener su título profesional, que es muy similar a nuestra realidad.

La recopilación de la información se hizo a través de llamadas telefónicas, realizadas a todos los egresados de la Facultad. Se aplicaron cuestionarios a los egresados matriculados en los programas de Diplomatura en Ciencias Empresariales y Licenciatura en Administración y Dirección de Empresas. El tamaño de la muestra fue de 224 estudiantes, de los cuales 67 eran Licenciados y el resto Diplomados.

Para los propósitos de esta investigación conviene precisar los conceptos de empleo y empleabilidad. Dicho de otra forma, ¿empleo es lo mismo que empleabilidad? Para aclarar lo que se entiende por ambos conceptos, tomamos como referencia lo mencionado por Manuel Fernández $z^{3}$ :

- Empleabilidad: una de las palabras clave de Bolonia.

- Se dice que la empleabilidad pervierte y degrada la educación superior.

- Pero empleabilidad es un concepto más completo y dinámico que empleo, puesto de trabajo o capacitación profesional

- Empleabilidad: significa desarrollar en el estudiante el potencial para conseguir trabajos y conservarlos. Es mucho más que obtener un título universitario (antes y ahora).
- El debate entre la orientación académica y la orientación profesional es un debate vacío en el ámbito universitario

- La universidad debe proporcionar un balance entre la formación técnica y científica para trabajos concretos, y otras competencias del universitario; todo junto es la base de la empleabilidad.

- La empleabilidad se inicia antes de la universidad, sigue durante las estudios universitarios, y se consolida a lo largo de la vida.

De modo que la empleabilidad es una categoría que trasciende lo meramente conceptual porque involucra competencias que el ser humano adquiere desde el entorno familiar pero que, producto de influencias de centros educativos donde estudió o aprendió -centro de educación inicial, colegio, universidad o trabajo-, las modula y perfecciona permanente para mantenerse vigente en el mercado laboral.

Dentro de ese contexto hablamos de empleabilidad como un mecanismo permanente de correspondencia del ser humano con el mercado laboral, que se caracteriza por exigir de las personas conocimientos actualizados, habilidades y destrezas para desempeñarse en diferentes puestos o realizando otras funciones, sin perder el nivel de profesionalidad; en suma, un nivel de competitividad que garantice de los egresados de cualquier universidad su polivalencia y capacidad de adaptarse a múltiples funciones a carta cabal.

Otra investigación que nos interesa citar fue la realizada por el profesor Raúl Porras Lavalle 4 . Porras examina las experiencias de universidades innovadoras, las propuestas y el esfuerzo en trabajos de investigación, formación profesional y extensión universitaria. Argumenta que los dos últimos chocan con un grave problema que afecta actualmente a América Latina. Según Porras, en el periodo de su investigación había en América Latina un estado fallido, dos estados en alto riesgo, cuatro estados en situación crítica y seis estados en situación comprometedora.

Para este autor, el Perú y los países en riesgo presentan un desarrollo desigual, altos índices de

2 Universidad Politécnica de Cartagena. (2004).

3 Fernández, Manuel. (2003).

4 Porras, Raúl. (2002). 
criminalidad, pérdida paulatina de la legitimidad del estado, degradación ambiental, violaciones de derechos humanos e instituciones desprestigiadas (Poder Ejecutivo, Poder Legislativo y Poder Judicial) y a punto de colapsar, y sólo les queda la universidad, reconocida por la ciudadanía como uno de los establecimientos más confiables.

Desde su creación, la universidad fue y es una institución prestigiosa y reconocida por las naciones y la ciudadanía. Al consolidarse los estados modernos en Europa, la universidad se constituye en una de las instituciones básicas de la sociedad, asignándosele como función principal la formación de recursos humanos necesarios para la administración gubernamental y la salud pública. Posteriormente, asume como responsabilidad la investigación científica y la extensión. La universidad latinoamericana, heredera de la universidad europea y al servicio de la corona española no obstante la reglamentación hispánica, contribuyó a la emancipación, enseñando a pensar críticamente a los criollos, independientemente de los textos de retórica y derecho canónico en ella utilizados. Conseguida la independencia, abre sus puertas al pensamiento liberal y revolucionario, rechaza la opresión, defiende a los indígenas, prepara a las élites para el poder, extiende sus servicios a la comunidad, se reforma a sí misma e investiga pese a la limitación presupuestal, y sobrepasa los pronósticos negativos para el siglo XXI.

La verdadera grandeza de la universidad latinoamericana no radica tanto en la investigación científica y tecnológica que medianamente realiza, sino en la persistencia heroica de sus profesores y estudiantes de propiciar el cambio que posibilite no sólo construir una universidad capaz de crear una cultura original y autónoma, sino que coadyuve en la formación de una sociedad más justa y humana.

Porras señala que la universidad peruana y latinoamericana es una institución autorizada por la ciudadanía, pese a las críticas y las crisis. Tiene serias dificultades, superables por cierto, especialmente en la formación de profesionales tal como lo describe en su investigación, pero que a la vez esta limitación le permitirá sobreponerse a las dificultades coyunturales. El diagnóstico que presenta resume la evolución de los estilos y la problemática de la formación profesional en el Perú, contrastada con las experiencias de universidades exitosas.

\section{OBJETIVOS DE LA INVESTIGACIÓN}

- Conocer a modo de diagnóstico los niveles y tipos de empleabilidad de la que gozan, en el mercado laboral, los egresados de la Facultad de Ciencias Administrativas (FCA) de la Universidad Nacional Mayor de San Marcos (UNMSM).

- Conocer el nivel de correspondencia que existe entre los planes de estudios de las escuelas profesionales de Administración, Negocios Internacionales y Turismo con las exigencias en términos de competencias laborales.

\section{HIPÓTESIS, VARIABLES E INDICADORES}

Para ello, se planteó como hipótesis de trabajo la siguiente:

"La empleabilidad de los egresados de la Facultad de Ciencias Administrativas de la UNMSM es concordante con el ámbito de ocupación laboral definido por la Universidad".

\section{VARIABLES}

Los variables e indicadores identificados para la presente investigación fueron los siguientes:

\section{Variable Independiente}

Ámbito de ocupación laboral definido en los planes de estudios por la Facultad.

\section{Indicadores}

- Planes de Estudios de las Escuelas de la FCA de la UNMSM.

- Perfil profesional.

- Nivel académico y experiencia profesional de los docentes.

\section{Variable Dependiente}

Nivel de empleabilidad de los egresados de la FCA de la UNMSM.

\section{Indicadores}

- Índice de logros profesionales.

- Tasa de empleo en el ámbito profesional.

- Tasa de empleo en otros ámbitos laborales.

\section{MÉTODOS Y RESULTADOS}

1. Metodología para el trabajo de campo. El universo de la investigación estuvo conformado por todos los bachilleres de la Facultad que 
al momento de realizar el trabajo de campo se encontraban tramitando sus expedientes para optar su título profesional, en el periodo correspondiente a marzo-octubre del 2007. Como el proceso de recopilación de datos se hizo a través de la Oficina de Trámite Documentario y la Oficina de Grados, Títulos y Convalidaciones de la Facultad, creemos que se abarcó -en la medida de lo posible- a todos los bachilleres, de allí que nuestra muestra inicial fue de 203 encuestados, de los cuales se han desechado 13 encuestas por estar incompletas o ser ilegibles; por tanto, el tamaño de la muestra (que comprendió las 190 encuestas restantes), garantiza la confiabilidad y credibilidad de sus resultados.

Adicionalmente, los resultados obtenidos fueron complementados con las entrevistas que se realizaron a algunos bachilleres elegidos en forma aleatoria, con el propósito de corroborar o precisar los datos iniciales.

2. Metodología para el procesamiento de datos. Las encuestas fueron procesadas a través del software aplicativo SPSS; previamente se elaboró una encuesta ad hoc. Ya para el diseño y presentación de los resultados fue necesario recurrir al software Microsoft Excel.

\section{ANÁLISIS Y DISCUSIÓN DE RESULTADOS}

El Gráfico $\mathrm{N}^{\circ} 1$ identifica la procedencia de los bachilleres que solicitan optar su Título Profesional. Así, los que provienen de la Escuela de Administración representan el 75\% del total, mientras que los egresados de las Escuelas de Negocios Internacionales y Turismo representan el $15 \%$ y $10 \%$ respectivamente.

Esta masiva solicitud de los bachilleres en Administración se explica por cuanto Negocios Internacionales y Turismo -por ser escuelas profesionales jóvenes- tiene muy pocos egresados a la fecha.

GRÁFICO No 1

ESCUELA A LA QUE PERTENECE.

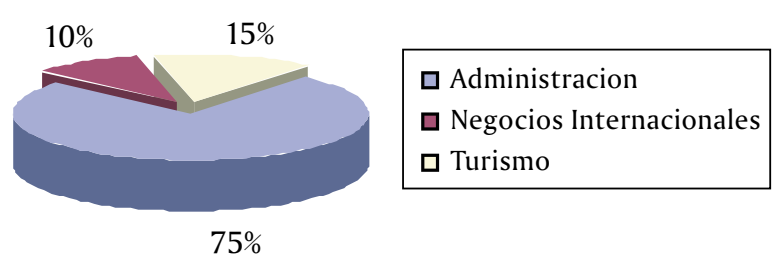

El Gráfico $\mathrm{N}^{\circ} 2$ está referido a medir el nivel de conocimientos que manifiestan haber tenido al momento de egresar de la universidad. Así el $1 \%$ de los encuestados indica haber contado con un nivel de conocimientos muy alto, el $46 \%$ indica haber egresado con un nivel alto de conocimientos, mientras que un $49 \%$ con un nivel de conocimiento medio y, por último, un $4 \%$ con bajo o muy bajo conocimientos necesarios para poder desarrollarse en el campo laboral.

Este dato es importante por cuanto nos permite contrastar lo que en términos sistémicos podemos tipificar como el producto final que la universidad pone a consideración del mercado laboral. Ello, en el mejor de los casos, indica que solo el $47 \%$ de los egresados habrían tenido conexión con el mundo laboral, mientras que el 53\% se habrían sentido desconectados con lo que exige el mercado, en términos de conocimientos.

En ese orden de ideas, otro aspecto de interés investigativo estuvo referido a conocer los planes de nuestros egresados luego de haber culminado sus estudios en la universidad; es lo que se conoce como las expectativas manifiestas que construyen las personas a partir de una competencia incorporada (estudios universitarios concluidos). Al respecto, un $59 \%$ de los encuestados manifestaron insertarse a laborar en una gran empresa, un $32 \%$ manifestaron que formarían su propia empresa y un $8 \%$ indicaron que viajarían al extranjero o trabajarían en una empresa familiar, entre otros, tal como se ilustra en el Gráfico $\mathrm{N}^{\circ} 3$.

Tales resultados llaman poderosamente la atención, por cuanto el $60 \%$ aspiraba trabajar en una gran empresa, en tanto que solo el $32 \%$ manifestaban querer embarcarse en un proyecto de empresa personal. Mas adelante, cruzando con los otros resultados, veremos que estos planes -en su mayoría- no se cumplieron.

Los siguientes gráficos (Gráficos $\mathrm{N}^{\circ} 4 \mathrm{y} \mathrm{N}^{\circ}$ 5) están dirigidos a conocer los sectores económicos donde nuestros egresados se encuentran

GRÁFICO No 2

NIVEL DE CONOCIMIENTOS AL EGRESAR

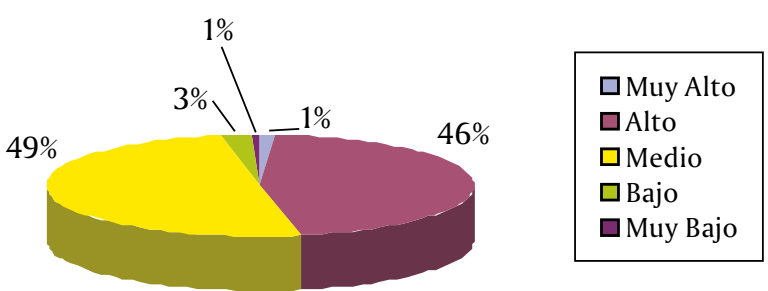




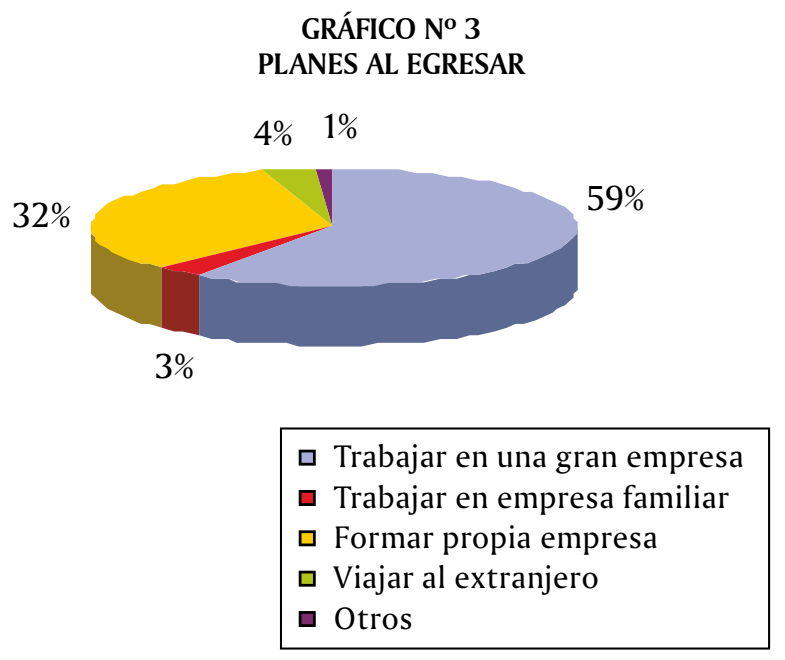

laborando. Los egresados, en primera instancia, laboran en empresas privadas (86\%) e instituciones públicas (14\%).

De los que manifestaron trabajar en empresas privadas, el $67 \%$ pertenecen al sector servicios, seguido de un $18 \%$ que lo hace en empresas del sector comercial, $5 \%$ industrial, $7 \%$ en exportación / importación y el resto (3\%), en otros sectores.

De los que manifestaron trabajar en el sector público, la muestra indicó que casi en su totalidad (91\%) corresponden al gobierno central, el 3\% en gobiernos regionales y gobiernos locales respectivamente, y el 3\% en otras instituciones públicas (ver Gráfico $\mathrm{N}^{\circ}$ 6).

GRÁFICO No 4

SECTOR DE LA EMPRESA DONDE LABORA

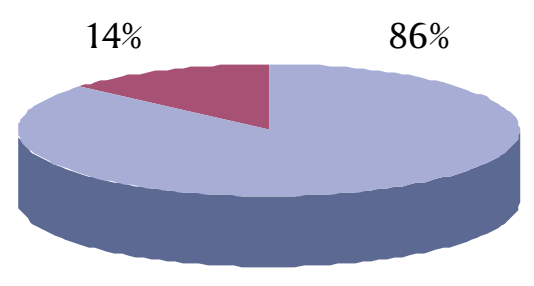

口Privada ๑Pública

GRÁFICO No 6

SECTOR DE LA EMPRESA PÚBLICA

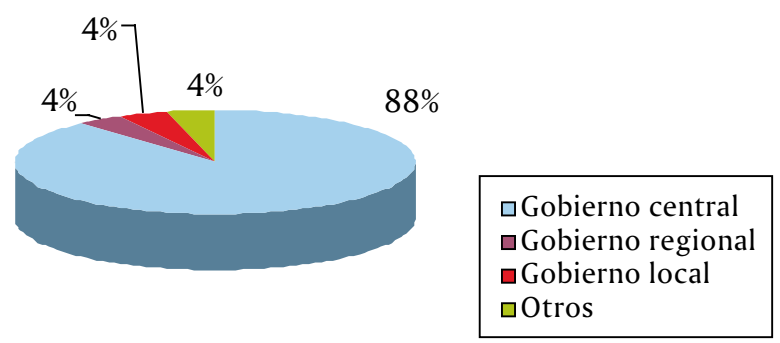

La relación contractual que mantienen los encuestados con sus empleadores es variada (Gráfico $\mathrm{N}^{\circ}$ 7). Por un lado, del total de los encuestados, los que han firmado un contrato a plazo indeterminado representan un $21 \%$, mientras que un $44 \%$ ha firmado un contrato a plazo fijo. Por otro lado, los contratados por servicios no personales representan un $10 \%$, en tanto que un $21 \%$ se encuentra en calidad de practicantes, y un $4 \%$ manifiestan tener otra modalidad de relación.

Otro dato de interés que es coherente con la relación contractual es el tiempo que viene laborando en el trabajo actual. Un 29\% manifestó una incorporación reciente pues contaban de tres a seis meses laborando en la empresa; un $16 \%$ indicó tener un año en la empresa como máximo, mientras que el $20 \%$ indicaba haber tenido a la fecha una permanencia de máximo dos años; el resto que constituyen el $35 \%$ tienen mas de dos años laborando en la misma institución.

De los que manifestaron encontrarse en calidad de practicantes (22\%), hay un $29 \%$ de ellos que vienen laborando recién desde hace seis meses. Tales hechos se explican porque un buen porcentaje de los encuestados egresaron de las aulas universitarias en el período de 2004 al 2006, situación que explicaría el alto porcentaje de practicantes.

Otro hecho de interés de la investigación estuvo referido a conocer los cursos, técnicas

\section{GRÁFICO No 5 SECTOR DE LA EMPRESA PRIVADA}

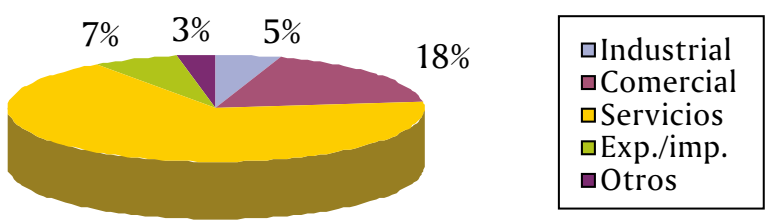

$67 \%$

GRÁFICO No 7 TIPO DE CONTRATO

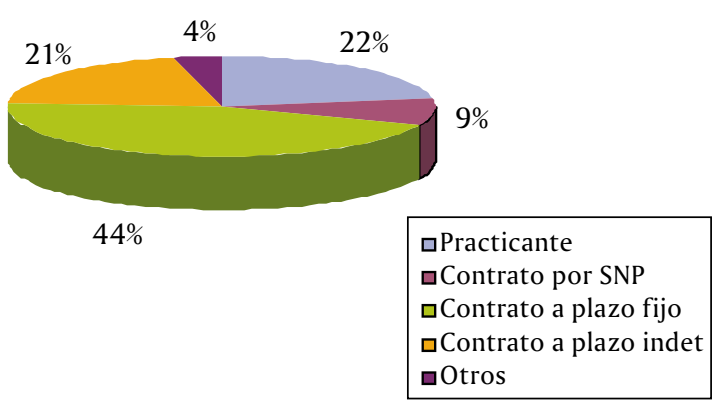


o instrumentos que se deben incorporar en el diseño de los nuevos planes de estudios en la Facultad, fundamentalmente para revertir las opiniones de aquellos que manifestaron haber tenido conocimientos medio, bajo o muy bajo al momento de egresar (Grafico $\mathrm{N}^{\circ} 8$ ). Las respuestas obtenidas son interesantes e innovadoras, a tomarse en cuenta al momento de incorporar nuevos cursos.

Como vemos en el grafico adjunto, el $42 \%$ sugirió cursos relacionados a la Administración General, con mayor énfasis en la práctica y modelos de gestión actuales y relacionados con la coyuntura económica y social. Los cursos sugeridos en este ítem fueron, por citar algunos: Incubadora de Negocios, Gestión de PYMEs, Modelos de gestión para empresas de servicios, E-Commerce, Gestión Pública, entre otros.

Un 16\% solicitó cursos relacionados a la especialización en Finanzas, un 12\% pidió cursos relacionados a los Negocios Internacionales, un $8 \%$ de la especialización en Marketing, un 5\% en cursos de Administración Turística, y un 4\% en especialización en Recursos Humanos y Logística, respectivamente. Solo un $14 \%$ de los entrevistados se abstuvieron de sugerir cursos.

Un aspecto importante que debemos añadir en esta parte de la investigación es el referido a la relación directa que guardan los cursos sugeridos con el ámbito o sector donde se desenvuelven los entrevistados, de modo que, a diferencia de los cursos que se dictan actualmente, tales cursos deben ser especializaciones por excelencia, que conduzcan a la obtención de Diplomados. Creemos que si la Universidad cumple con diseñar y ofertar cursos con tales características, estará potenciando la profesionalidad y competitividad de nuestros egresados en el mercado laboral.

Así, creemos que las competencias laborales sugeridas por los encuestados se organizan de la siguiente manera:

- Básicas. Se refiere a aquellas de índole formativa que requiere el egresado para desempeñarse

GRÁFICO N ${ }^{\circ} 8$

ÁMBITO DE LOS CURSOS SUGERIDOS

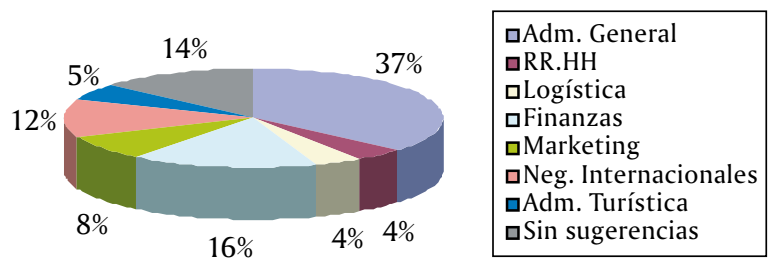

en cualquier actividad productiva, tales como la aplicación de sistemas de procesamiento de datos, interpretación de la realidad económica, aplicar sistemas numéricos para análisis de datos, saber expresarse y saber escuchar.

- Genéricas. En este tipo de competencias hemos registrado aquellos conocimientos y habilidades que están asociadas al desarrollo de diversas áreas y sub áreas ocupacionales; es decir, son las competencias que definen un perfil concreto para las distintas actividades. Por ejemplo, analizar y evaluar información, trabajar en equipo, planear acciones, coaching, deontología, liderazgo, desarrollo emocional, manejo de personal, adaptación, entre otras.

- Específicas. Se refieren a aquellas competencias asociadas a conocimientos y habilidades de índole específica y que son necesarias para la ejecución de su especialidad. Por ejemplo, para aquellos que estudiaron Administración en Turismo y/o Negocios Internacionales, los encuestados han considerado el dominio mínimo de dos idiomas, otros han considerado la metodología de investigación, formulación de proyectos o planes de negocio, formación de consultorías, etc; todo ello en el entendido de que estos cursos potenciarán aún más sus competencias profesionales. Después de todo, creemos que la formación universitaria debe tender precisamente a la incorporación y afinamientos de las competencias específicas.

En relación a las competencias genéricas -importantes para cualquier tipo de desempeño laboral- podemos apreciar en el Gráfico $\mathrm{N}^{\circ} 9$ que un $50 \%$ de los entrevistados sugieren potenciar las competencias de este tipo, referidas al desarrollo de trabajo en equipo, trabajo bajo tensión, incorporación y modulación de valores terminales e instrumentales, etc., que permitan un mejor desempeño en sus labores. Como contraparte, solo un $24 \%$ prefirió no emitir opinión al respecto.

\section{COMPETENCIAS SUGERIDAS}

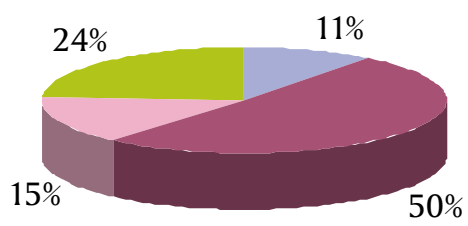

口Básicas 口Genéricas 口Específicas $\square$ Abstenciones 
1. Demostración de la Hipótesis. Como quiera que toda investigación con estas características exige la debida demostración, fue necesario recurrir a la Estadística para comprobar si existe correlación entre las variables planteadas.

Cuadro No 1

Variables introducidas/ eliminadas (b)

\begin{tabular}{|c|c|c|c|}
\hline Modelo & $\begin{array}{c}\text { Variables } \\
\text { introducidas }\end{array}$ & $\begin{array}{c}\text { Variables } \\
\text { eliminadas }\end{array}$ & Método \\
\hline 1 & $\begin{array}{c}\text { Nivel de cono- } \\
\text { cimientos al } \\
\text { egresar, Planes } \\
\text { de desarrollo } \\
\text { profesional (a) }\end{array}$ & Introducir \\
\hline
\end{tabular}

Donde:

a) Todas las variables solicitadas introducidas.

b) Variable dependiente: logros alcanzados en la actualidad.

Resumen del modelo
\begin{tabular}{|c|c|c|c|c|}
\hline Modelo & $\mathbf{R}$ & $\begin{array}{c}\mathbf{R} \\
\text { cuadrado }\end{array}$ & $\begin{array}{c}\text { R cuadrado } \\
\text { corregida }\end{array}$ & $\begin{array}{c}\text { Error típico de } \\
\text { la estimación }\end{array}$ \\
\hline 1 &, $977(\mathrm{a})$ &, 955 &, 955 &, 128 \\
\hline
\end{tabular}

Donde:

a) Variables predictoras: (constante), nivel de conocimientos al egresar, planes de desarrollo profesional.

1.1. Interpretación del Coeficiente de Correlación y de Determinación

$$
\mathrm{R}=0.977
$$

El 97.70\% representa la correlación que existe entre los logros alcanzados en su desarrollo profesional con el nivel de conocimientos y los planes de desarrollo al egresar.

$$
\mathrm{R}^{2}=0.955
$$

Los logros alcanzados tienen una variación del $95.50 \%$ debido al nivel de conocimientos y los planes de desarrollo al egresar.

Cuadro $N^{\circ} 3$

Coeficientes (a)

\begin{tabular}{|c|l|l|l|l|l|l|}
\hline Modelo & & \multicolumn{2}{|c|}{$\begin{array}{c}\text { Coeficientes no } \\
\text { estandarizados }\end{array}$} & \multicolumn{1}{|c|}{$\begin{array}{c}\text { Coeficientes } \\
\text { estandarizados }\end{array}$} & t & Sig. \\
\hline & & B & Error típico & Beta & & \\
\hline 1 & (Constante) &, 466 &, 043 & & 10,913 &, 000 \\
\hline & $\begin{array}{l}\text { Planes de desarrollo } \\
\text { profesional }\end{array}$ &, 542 &, 008 &, 977 & 65,142 &, 000 \\
\hline & $\begin{array}{l}\text { Nivel de conocimientos } \\
\text { al egresar }\end{array}$ & $-2,242 \mathrm{E}-03$ &, 015 &,- 002 &,- 147 &, 884 \\
\hline
\end{tabular}

Donde:

a) Variable dependiente: logros alcanzados en la actualidad.

Cuadro No 4
\begin{tabular}{|l|l|l|l|l|l|c|}
\hline \multicolumn{1}{|c|}{ ANOVA (b) } \\
\hline Modelo & & $\begin{array}{c}\text { Suma de } \\
\text { cuadrados }\end{array}$ & gl & $\begin{array}{c}\text { Media } \\
\text { cuadrática }\end{array}$ & F & Sig. \\
\hline 1 & Regresión & 69,420 & 2 & 34,710 & 2123,444 &, $000($ a) \\
\hline & Residual & 3,269 & 200 &, 016 & & \\
\hline & Total & 72,690 & 202 & & & \\
\hline
\end{tabular}

Donde:

a) Variables predictoras: (constante), nivel de conocimientos al egresar, planes de desarrollo profesional.

b) Variable dependiente: logros alcanzados en la actualidad. 
1.2. Análisis de Existencia de la Regresión.

$\mathrm{Al}$ analizar los niveles de significación de cada una de las variables independientes, la mayoría de las variables son menores a 5\%, a excepción del nivel de conocimientos al egresar; sin embargo, podemos seguir afirmando la existencia de la regresión múltiple, por cuanto basta que sólo una de ellas sea menor a $5 \%{ }^{5}$

1.3. Análisis de la existencia del Modelo Global.

El análisis de varianza se realiza en este caso para determinar si existe o no regresión lineal en la población de la variable dependiente "logros alcanzados en la actualidad" con las variables independientes reflejadas en "nivel de conocimientos y planes desarrollo al egresar".

Prueba de hipótesis para el análisis global de los coeficientes de regresión:

Formulación de la hipótesis:

$$
\mathrm{H}_{0}: \mathrm{B}_{1}=\mathrm{B}_{2}=0
$$

$\mathrm{H}_{1}$ : Necesariamente una de las $B_{i}$ debe ser diferente de 0

Prueba de significancia:

$\mathrm{a}=0.05$

$1-\mathrm{a}=0.95$

$\mathrm{n}=190$

Como queremos analizar la existencia del modelo global, con los grados de libertad 2,200 en la tabla de probabilidades de F, se encuentra el valor crítico $\mathrm{F}_{(2,190)}$. Debido a que no se encuentra el mismo valor en la tabla, tomamos $\mathrm{F}_{(2,200)}$, con un nivel se significancia del 5\%.

1.4. Regla de Decisión.

Se rechaza la $\mathrm{H}_{0}$

$\mathrm{F}_{\mathrm{c}}>3.04$

\section{Cálculo del estadístico}

De la tabla del ANOVA el $\mathrm{F}_{\mathrm{c}}=2123.44$

\subsection{Conclusiones.}

Se rechaza $\mathrm{H}_{0}$ y se acepta $\mathrm{H}_{1}$

En consecuencia, a partir de tales resultados de la prueba global, podemos inferir que por lo menos uno de los coeficientes de regresión poblacional es diferente de 0 , y que existe regresión global de logros alcanzados con el nivel de conocimientos al egresar y con los planes de desarrollo profesional.

\section{CONCLUSIONES Y RECOMENDACIONES}

1. A partir de los resultados obtenidos podemos concluir que existe una mediana correspondencia entre el ámbito de ocupación laboral definido por la Universidad, a través de sus planes de estudios, y el nivel de empleabilidad y ocupabilidad de nuestros egresados en el mercado laboral. Tal afirmación se puede corroborar cuando el 53\% de los encuestados manifestaron que los conocimientos impartidos en la UNMSM sirvieron poco o medianamente para su desempeño laboral óptimo. Ello implica que la Universidad debe mantener una comunicación estrecha con los stakeholders, a fin de saber cuáles son sus requerimientos en términos de recursos profesionales, para incorporarlos en sus futuros planes de estudios.

2. Asimismo, la investigación también demuestra que un porcentaje representativo de nuestros egresados tienen empleos en posiciones inferiores de la estructura organizacional, cumpliendo funciones administrativas y operativas nada o poco gravitantes para la organización. Esta situación se puede explicar desde 2 posiciones: a) Que los encuestados fueron en su mayoría egresados de los últimos años (2004-2006), que recién empiezan a laborar profesionalmente y es obvio que lo harán desde abajo, y b) Que los planes de estudios no guardan relación con lo que exige el mercado laboral, por lo que nuestros egresados sistemáticamente son postergados de ascender a funciones o puestos relevantes, dado que no se encuentran debidamente preparados para ello. Contrastando y cruzando otros datos, podemos afirmar que los empleos poco relevantes de nuestros egresados se explican por la primera posición, lo que a su vez demuestra un elemento incontrolable en la investigación.

3. En ese orden de ideas, la preocupación por la empleabilidad y ocupabilidad de nuestros egresados debe ser uno de los objetivos principales de la Facultad. Ello implica que al momento de reestructurar los planes de estudios, en la fase de diagnóstico, se deben tomar muy en cuenta las opiniones y sugerencias que emitan

5 Se está trabajando bajo un nivel de confianza del 95\% $(1-\mathrm{a}=0.95)$. 
los líderes y directivos de las organizaciones privadas o públicas.

4. Debemos entender que la empleabilidad es un concepto mucho más dinámico que empleo, puesto de trabajo o capacitación profesional. Trabajar por la empleabilidad de nuestros egresados implica potenciar sus competencias para conseguir trabajos y conservarlos. Esto es mucho más que el proceso que conduce solo a la obtención del título profesional.

5. En ese contexto la universidad debe proporcionar un balance entre la formación técnica y científica para trabajos especializados, sin descuidar la formación del ciudadano que se integra a la sociedad para trascender en el tiempo. Postulamos en suma a una empleabilidad con formación integral del hombre, que conjugue lo conceptual con lo procedimental $\mathrm{y}$ con lo actitudinal fundamentalmente.

\section{BIBLIOGRAFIA}

Espacio Europeo de Educación Superior (EEES). (2006). El reto de la Convergencia Europea en la UPCT. Caminando hacia el 2010. España, EEES, 16 y 17 febrero del 2006.

Espinoza, Nemesio. (2004). "Las aptitudes y actitudes empresariales de los estudiantes de administración en las universidades públicas de Lima - Callao”. En: Revista Gestión en el Tercer Milenio. Lima, Facultad de Ciencias Administrativas de la UNMSM, noviembre 2004.

Fernández, Manuel. (2003). El reto de la convergencia europea en la UPCT. Caminando hacia al 2010. Madrid, Espacio Europeo de Educación Superior (EES).

Fernández, Manuel. (2005). Qué no es el EEES. Siete pifias del proceso de Bolonia. España, Xunta de Galicia.
Porras, Raúl. (2007). Notas para el estudio de la Formación Profesional en Perú. Lima, Universidad Nacional Federico Villarreal.

Universidad Politécnica de Cartagena. (2004). Caracterización y empleabilidad de los egresados de la Facultad de Ciencias de la Empresa. Bogotá, Decanato de la Facultad de Ciencias de la Empresa, versión preliminar de septiembre del 2004.

\section{INTERNET}

Erosa, Victoria. (2007). “Competencias Profesionales del área de Administración: La perspectiva de estudiantes, profesores, egresados y empleadores- El caso de México". México, Universidad Juárez Autónoma de Tabasco, julio 2007. En: http://www.6x4uealc.org/ site2008/p01/14.pdf.

García, Juan y Pérez, Carmen. (2008). "Competencias profesionales y empleabilidad". España, Espacio Europeo de Educación Superior (EEE), Universidad Europea Miguel de Cervantes, setiembre 2008. En: http://www. rieoei.org/deloslectores/2444Manjon.pdf

García, María y Vico, Antonio. (2008). "La formación universitaria en Administración y Dirección de Empresas. Análisis de su adaptación al mercado de trabajo y propuesta del Plan de Estudio". España, abril 2008. En: http://www.aeca.es/pub/monog/formacionuniversitaria.htm

Piscoya, Luis. (2008). "Formación Universitaria vs. Mercado Laboral II". Lima, Fondo Editorial Universidad Nacional Mayor de San Marcos, noviembre 2008. En: http://www.infomediarios.com/2008/11/18/libro-formacionuniversitaria-vs-mercado-laboral-iil- 